\title{
VON EINER NEUEN POETISCHEN KONZEPTION BEI EURIPIDES
}

\begin{abstract}
Summary: Euripides' Helen was performed in 412, some months after the complete débâcle of the Athenian army on Sicily. Aristophanes speaks of the "new Helen", and there is indeed no reason to criticize the pretty heroine. This new character corresponds to the new poetic conception Euripides presents in the Helen as in the contemporary Andromeda, too, after having tried to apply it already in the Iphigenia Taurica (414 or 413). In the mentioned tragedies large-scale operations as the Trojan War are missed. However, this war is condemned in the diccussions of the post-war time in Helen. In the action prevail rather single fates and a lot of adventures. To return home proves to be a remarkable motive, when according to Thucydides only few succeeded in escaping from Sicily. The hard struggle to save the life against tyrannical violence in far-off barbarous countries seem to be important for the new conception. Cheerful components are the love and the happy ending. But there is no doubt: The new poetic manner does not prevent tragedies from remaining tragedies.
\end{abstract}

Key words: Euripides' Helen, Andromeda, Iphigenia Taurica, Athens, polisdemocracy, Sicily excursion

Die Nachricht von der vernichtenden Niederlage, in der die athenischen Streitkräfte 413 bei ihrem groß angelegten Einsatz auf Sizilien völlig aufgerieben wurden (vgl.

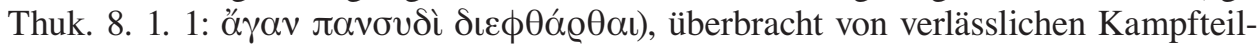
nehmern, die sich retten konnten und wahrheitsgetreu berichteten - diese Nachricht wurde in Athen zunächst weitgehend bezweifelt. So beginnt Thukydides das achte Buch seines Geschichtswerkes, nachdem er im Vorhergehenden die voller Zuversicht oder eher Hybris - unternommene Sizilienexpedition analysiert hat, die mit dem Ziel,

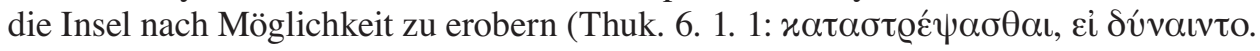
Vgl. 6. 6. 1; 7. 75. 7), ein Ausdruck athenischer Machtpolitik war.

Nach Erkenntnis des tatsächlich Geschehenen gerieten diejenigen unter Druck, die das Sizilienunternehmen unterstützt und die Hoffnung erweckt hatten, die Insel in

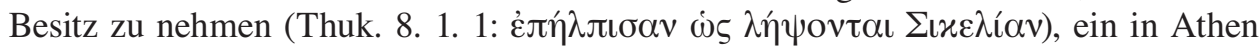


lang gehegter Wunsch (Thuk. 3. 86; 6. 1. 1). Dabei hatte die attische Volksversammlung Anfang Sommer 415 selbst für dieses Unternehmen gestimmt (Thuk. 8. 1. 1; 6. 8. 1 f.). In einer zweiten Ekklesia fünf Tage später wurde überdies die schnelle Ausrüstung der Schiffe für die Sizilienoperation beschlossen (Thuk. 6. 8. 3). So konnte eine sehr kampfkräftige athenische Flotte Mitte Sommer 415 zum Einsatzgebiet aufbrechen in der Hoffnung auf Machterweiterung (Thuk. 6. 30 f.), um am Ende in ein völliges Desaster zu geraten (Thuk. 7. 87. 5 f.). Euripides hatte an den Dionysien 415 mit seinen Troerinnen vergeblich gewarnt.

Die athenischen Invasoren fuhren in den Untergang, den sie sich im Grunde selbst bereitet haben. Das Scheitern auf Sizilien wirkte sich erheblich auf den Verlauf des Peloponnesischen Krieges aus. Attische Bundesgenossen wie Chios, Mytilene und Methymna auf Lesbos, Euböa und Weitere fielen von der führenden Polis ab, und den Spartanern gelang es, von Persien Gelder für den Kampf gegen Athen zu erhalten. Die Entwicklung, den großen Krieg zu verlieren, schien sich auf diese Weise für die machtbewusste Polisdemokratie abzuzeichnen. ${ }^{1}$

Nur wenige Monate nach dem athenischen Fiasko auf Sizilien - es spielte sich im Hochsommer bis Herbst $413 \mathrm{ab}^{2}$ - überraschte Euripides sein Publikum in Athen an den Dionysien 412 mit der Helena. ${ }^{3}$ Es war die „neue Helena“, wie es bei Aristo-

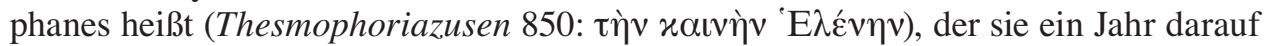
glänzend parodierte. Die schöne Tochter des Zeus wurde auch in dem euripideischen Novum viel bewundert, doch zum Schelten bestand hier keine Veranlassung. Helena war weit davon entfernt, ihre Ehe zu brechen. Sie wurde von Hermes nach Ägypten zu Proteus, dem dortigen Landesherrscher, in Sicherheit gebracht. Die Helena aber, die Paris entführte und um die der Trojanische Krieg entbrannte, war ein von Hera aus

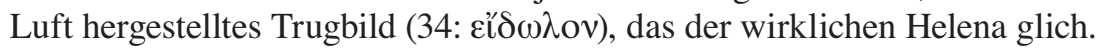

Der neuen Helena entspricht die neue poetische Konzeption, von der Euripides 412 bei der Helena und der im gleichen Jahr aufgeführten Andromeda ${ }^{4}$ ausging. Bereits 414 oder 413 scheint sie der Dichter mit der Iphigenie bei den Taurern ${ }^{5}$ versucht

\footnotetext{
${ }^{1} \mathrm{Zu}$ den historischen Bedingungen vgl. BENGTSON, H.: Griechische Geschichte von den Anfängen bis in die römische Kaiserzeit. 7. Auflage. München 1986, 208-225; WILCKEN, U.: Griechische Geschichte im Rahmen der Altertumsgeschichte. Berlin 1958, 180-193.

2 Bengtson (Anm. 1) 555.

${ }^{3}$ Ausgaben: Euripidis Helena. Edidit K. Alt. Leipzig 1964. Euripidis fabulae. Edidit J. DiGGLE. Vol. III. Oxford 1994.

Kommentare: Euripides, Helena. Herausgegeben und erklärt von R. KANNICHT. Bde I-II. Heidelberg 1969. Euripides, Helen. With introduction, translation and commentary by P. BURIAN. Oxford 2007. Euripides, Helen. Edited by W. Allan. Cambridge 2010. Sekundärliteratur: PIPPIN, A. N.: Euripides' Helen, a Comedy of Ideas. Classical Philology 55 (1960) 151-163; ZunTZ, G.: On Euripides' Helena. Theology and Irony. In Entretiens sur l' antiquité classique 6. Vandoeuvres-Genève 1960, 199-241. MATTHIESSEN, K.: Elektra, Taurische Iphigenie und Helena. Untersuchungen zur Chronologie und zur dramatischen Form im Spätwerk des Euripides [Hypomnemata 4]. Göttingen 1964. LESKY, A.: Die tragische Dichtung der Hellenen. 3. Auflage. Göttingen 1972, 413-425. WRIGHT, M.: Euripides' Escape Tragedies: A Study of "Helen", "Andromeda" and "Iphigenia among the Taurians". Oxford 2005.

${ }^{4}$ PAgAno, V.: L' Andromeda di Euripide. Edizione e commento dei frammenti. Alessandria 2010.

${ }^{5}$ KYRIAKOU, P.: A Commentary on Euripides' Iphigenia in Tauris. Berlin 2006. Hall, E.: Adventures with Iphigenia in Tauris. A Cultural History of Black Sea Tragedy. Oxford 2013.
} 
zu haben. Es geht dabei nicht mehr um Großereignisse wie den Trojanischen Krieg, sondern eher um einzelne Schicksale und Auseinandersetzungen abenteuerlicher Art. Auch sie sind mit Gefahren verbunden und nicht weniger tragisch, wenn sie die Kräfte des Einzelnen $\mathrm{zu}$ extremen Leistungen herausfordern, um sich gegen herrschende feindliche Gewalten zu behaupten.

Der Trojanische Krieg findet sich indessen keineswegs ausgeblendet. Er ragt von fern immer noch in seinen abschreckenden Auswirkungen herein, aber er ist nicht mehr die Grundlage des Spieles, auch wenn er verständlicherweise in der Nachkriegszeit der Helena zu häufigen Debatten führt. So gleich am Anfang des Dramas im Gespräch zwischen Helena und dem Trojakämpfer Teukros, der sich als einer der Achaier vorstellt,

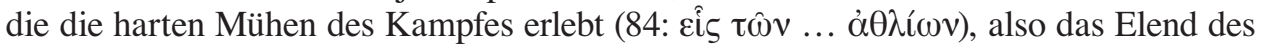
Krieges gesehen haben. Er war selbst an der Zerstörung Trojas beteiligt und hat sich

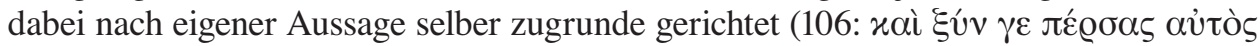
$\dot{\alpha} v \tau \alpha \tau \omega \lambda \hat{\mu} \mu \eta v)$. Am Ende, als es wieder ums Leben geht, beim riskanten Ausbruch aus Ägypten, fordert Menelaos, einst an der Spitze des griechischen Heeres vor Troja (395 f.) und auch ein Zerstörer (401 f., 806; vgl. auch 1493 f.), seine überlebenden (vgl. 397-399)

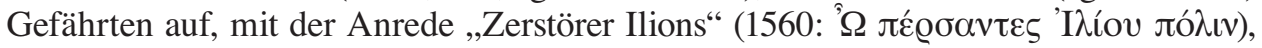
nach griechischer Sitte zuzugreifen (1561: $\alpha v \alpha \varrho \tau \alpha ́ \alpha \alpha \nu \tau \varepsilon \varsigma)$, und seine schöne Gattin stimmt, als die Griechen über die Barbaren (1604) herfallen, ähnlich kühn mit ein, wenn sie an den vor Troja gewonnenen „Ruhm“ (1603: $x \lambda$ ćo૬) erinnert (vgl. 808, 845).

Vorher schon bekannte Helena, dass ihre reizende Gestalt die Burg Dardaniens vernichtet hat und die Achaier (383-385); aber die sind schon vernichtet (385:

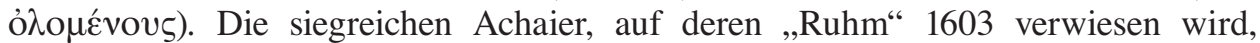
erscheinen hier doppelt zugrunde gegangen.

In einer Reihe von Versen, in der gezeigt wird, dass Sieger wie Besiegte in die gleiche Misere des Todes geraten (38-40 und 52 f., 109 f., 362 f. und 370 f., 383-385, 608-610), erfährt der Trojanische Krieg in der Helena aus kritischer Sicht eine scharfe Ablehnung. Das gilt ebenso für eine weitere Gruppe von Aussagen dieser Tragödie zu den Folgen des Krieges (362-374, 1107-1116, 1122-1131, 1151-1156, 1159). So unterlässt es der Dichter auch in der Helena nicht, seine Stimme gegen den Unsinn des Krieges zu erheben ${ }^{6}$ - die Katastrophe der athenischen Sizilienoperation wenige Monate vor der Helena-Aufführung war noch sehr aktuell.

Zur neuen Thematik, die den abenteuerlichen Verlauf der Handlung plausibel machen will, gehört die Ferne, in der, weit von Griechenland, also in Ländern von Barbaren, ein tyrannischer Herrscher eine unbegrenzte Macht hat. In der Iphigenie bei den Taurern ist es Thoas, der alle ankommenden Fremden töten lässt, und in der Helena verfährt der in Ägypten regierende Theoklymenos mit eintreffenden Griechen in gleicher Weise.

Mit den Gefahren, die aus der fernen Welt voller Abenteuer hervorgehen, verbindet sich in der tragischen Neuentwicklung überdies ein oft wiederkehrendes Motiv. Es ist der ständige und oft erbitterte Kampf, sich nach Hause durchzuschlagen und die griechische Heimat wieder zu erreichen - also ein Stück Odyssee.

${ }^{6}$ KuCH, H.: Glücklose Initiative: Euripides, ,Helena“ 1151-1156, 1159. Das Altertum 59 (2014) 19-21. 
Es fällt auf, in welchem Maße die Helena das Thema der Rückkehr nach Griechenland aufgreift, das 412 offenbar sehr aktuell gewesen sein muss angesichts der Vielzahl der Fälle, in denen eine Heimkehr nach der eklatanten Niederlage auf Sizilien nicht mehr möglich war. Mit der athenischen Kriegspolitik ging eine auch zahlenmäBig starke Armee verloren. Wer nicht den Tod fand, wurde gefangen genommen und in die Steinbrüche von Syrakus geworfen, ein Teil versklavt. Nur Wenigen gelang die

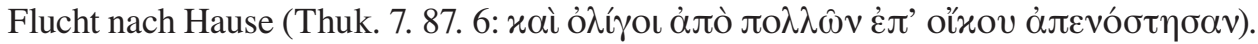
Kein Geringerer als Thukydides hat über die Folgen der Sizilienoperation berichtet (7. 85-87).

Die Euripideische Tragödie ist indessen kein Geschichtswerk. Wenn sie auch auf ihre Zeit einzugehen scheint, bleibt sie in jedem Fall tragisches Kunstwerk und sieht ohne Frage und unbedingt ihre Aufgabe darin, mit den Mitteln des tragischen Genos zu arbeiten. So lässt sich vielleicht denken, dass der Dichter unter dem Eindruck der schlimmen sizilischen Tatsachen mit seiner neuen Spielweise im Bereich des Mythos nach Möglichkeiten gesucht hat, sich aus verzweifelter Lage durchzusetzen und nach Hause zurückzukehren.

Helena nennt schon im Prolog ihrer gleichnamigen Tragödie ihre „,nicht namen-

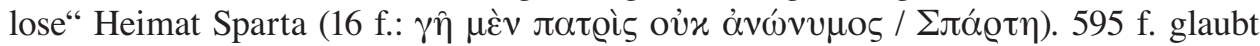

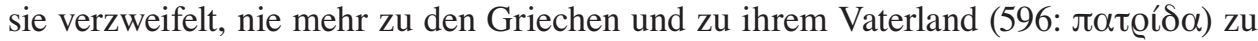
gelangen.

Unter den Rückkehrern in der Helena finden sich paradigmatisch auch zwei Trojakämpfer. Teukros will auf der Rückfahrt von der zerstörten Stadt nach Kypros gelangen, das ihm Apollon als Wohnsitz und neue Heimat zugewiesen hat (148-150), und Menelaos scheint zunächst die Rückkehr ins Vaterland (407: غ̇ऽ $\pi \alpha ́ \tau \varrho \alpha v$, ein Wort, das hier nach 402 und 405 das dritte Mal begegnet) nicht zu glücken. Wie der Chor, gestützt auf die Weissagung der Theonoe, mitteilt, hat Menelaos noch nicht die Häfen

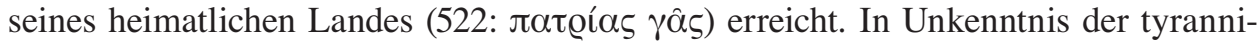
schen Verhältnisse in Ägypten versteht er nicht, weshalb es ihm nicht möglich sein soll,

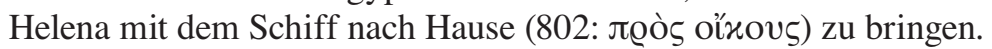

Theonoe bedauert seine Strapazen und tröstet ihn, der nicht weiß, ob er nach

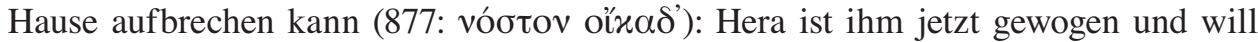

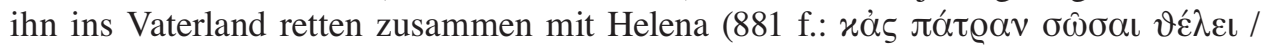

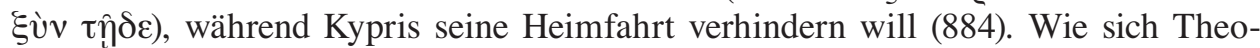
noe entscheiden wird, bleibt vorerst noch offen (887-891). Nachdem Helena (929) und Menelaos (990) jedoch vehement und überzeugend für ihre Rettung plädiert haben, stimmt ihnen die Seherin auf ihre Weise zu (1024-1027), ausgehend von Ehrfurcht vor

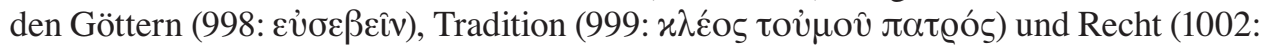

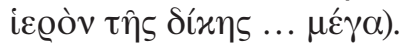

So können Helena und Menelaos ihre Rettungsaktion vorbereiten, um ins Vater-

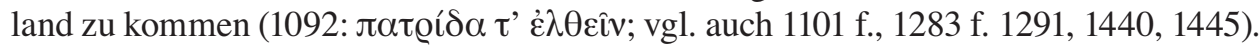

Der Aufbruch nach Griechenland wird vom Chor mit seinem Lied begleitet. Bevor das rettende Schiff in See sticht, fordert der Chor die Matrosen auf, Helena nach Mykene und Sparta zu bringen (1462-1477), und er wünscht sich selber, sich auf Vogelschwingen erheben zu können, um hinwegzufliegen (1478-1486; vgl. 1388 f.). Die 
Vögel werden aufgerufen, die Botschaft zum Eurotas, also zur Peloponnes, zu tragen,

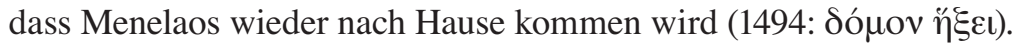

Aber da ist erst noch ein erbitterter Kampf auf dem auslaufenden Schiff gegen die barbarische Mannschaft zu bestehen, nach der geglückten Intrige, die Helena gegen den verliebten Theoklymenos betrieb, um die Rückfahrt zu sichern. Menelaos ruft zu Beginn der Auseinandersetzung Poseidon und die Nereiden an, ihn zu den Küsten von Nauplia in Sicherheit zu bringen (1586 f.), d. h. zur Peloponnes, und befiehlt, das Schiff nach Griechenland zu lenken (1611).

Der getäuschte Theoklymenos würde gern gewaltsam eingreifen, doch die Dioskuren halten ihn zurück. Sie werden ihre Schwester Helena zum Vaterland (1665: $\pi \alpha ́ \tau \varrho \alpha v)$ begleiten und selbst der Barbarenherrscher gibt am Ende seine Zustimmung zu Helenas Fahrt nach Hause (1683: $\pi$ @òs oîxov).

Eine wichtige, ja unverzichtbare Komponente des neuen Euripideischen Spiels bildet, wie sich bereits zeigte, die Liebe. Es ist in der Helena der Reiz tiefer Liebe zwischen lang Getrennten - ein Thema, das so feinfühlig gestaltet wird, dass Helena und Menelaos beinahe idealisiert werden. Dass sich indessen gerade der herrschende barbarische Gewaltmensch in die griechische Heldin der Tragödie verliebt, ist ein besonderer Effekt der Handlung. Eine Gegenliebe kann da nicht aufkommen. So werden Mittel eingesetzt, um einer Barbarenehe zu entgehen.

Mit weiblicher List vor allem und der ohne sonderliche Bedenken eingesetzten Kunst der Intrige gelingt es der Helena - wie schon der Iphigenie - sich zu retten und in einer risikoreichen Aktion am Ende wieder das Heimatland zu gewinnen. Dabei ist das tapfere Eingreifen des Orest und Pylades in der Iphigenie bei den Taurern und des Menelaos in der Helena nicht zu übersehen, das eine Voraussetzung für den Erfolg schafft. Auch die lenkenden Götter spielen hier mit. So schließen die Iphigenie bei den Taurern und die Helena mit einem Happy Ending. Das trifft ebenfalls auf die Andromeda nach der Rettung durch Perseus zu.

Es besteht jedoch kein Zweifel, dass die so gestalteten Dramen bei allen Neuerungen Tragödien bleiben. Der Weg der Euripideischen Konzeption führt später zu Menander.

Heinrich Kuch

Berlin

heinrich.kuch@t-online.de 
